\title{
Metodología de mejoramiento en el desempeño de sistemas de producción. Aplicaciones en Pymes de la confección
}

\section{Methodology improvement in the performance of production systems. Applications in SMEs confection}

\author{
María A. Solano*, Juan J. Bravo*, Jaime A. Giraldo** $§$ \\ * Facultad de IngenieríaEscuela de Ingeniería Industrial y Estadística, \\ Universidad del Valle, Santiago de Cali, Colombia. \\ ** Departamento de Ingeniería Industrial, Universidad Nacional de Colombia, \\ Manizales, Colombia.
}

§alexasolanoce@hotmail.com,juan.bravo@correounivalle.edu.co,jaiagiraldog@unal.edu.co

(Recibido: Abril 25 de 2011-Aceptado: Noviembre 19 de 2012)

\begin{abstract}
Resumen
Se presenta una metodología de mejoramiento en el desempeño del sistema de producción en Pymes de la confección, consistente en la utilización de técnicas multicriterio para la selección y jerarquización de las prioridades competitivas a las que el sistema debe responder; el empleo de un procedimiento general para evaluar la coherencia estructural de la estrategia de operaciones en términos de las prioridades competitivas, los sistemas de producción y las palancas de fabricación; y finalmente la modelación del sistema de producción mediante simulación de eventos discretos con el fin de experimentar diversas alternativas de mejoramiento que estén alineadas con las prioridades competitivas más relevantes. Como resultado se aplicó la metodología propuesta a una muestra de cinco Pymes del sector de la confección de la ciudad de Manizales (Colombia) en su primera etapa, encontrándose que las prioridades competitivas calidad y tiempo de entrega son las más relevantes. Seguidamente a la Pyme con el peor desempeño se le aplicaron las demás etapas, demostrándose finalmente la utilidad de la metodología al lograr variar el desempeño de su sistema de producción en términos de aumentar un indicador de efectividad de un valor de 2.12 a 2.32 en una escala de 0 a 5 , mejorando su desempeño en cerca de un $10 \%$.
\end{abstract}

Palabras clave: Prioridades competitivas, Pymes de confección, Simulación de eventos discretos, Sistemas de producción

\begin{abstract}
.
This paper presents a methodology for improving the performance of production system in confection SMEs consisting in the use of multicriteria techniques for the selection and prioritization of competing priorities to which the system should respond, the use of a general procedure evaluate the structural coherence of the strategy of operations in terms of competitive priorities, production systems and manufacturing levers, and finally the production system modeling using discrete event simulation in order to experiment improvement alternatives that are aligned with the most important competitive priorities. As a result the proposed methodology was applied to a sample of five SMEs in the manufacturing sector of the city of Manizales in the first stage, finding that the competitive priorities of quality and delivery time are the most relevant. Next to the worst-performing SMEs were applied to the other stages, showing finally the usefulness of the methodology to achieve performance vary their production system in terms of raising an indicator of effectiveness of a value of 2.12 to 2.32 on a scale 0 to 5 , to improve its performance by about $10 \%$.
\end{abstract}

Keywords: Competing priorities, Discrete event simulation, Production systems, SMEs clothing 


\section{Introducción}

Para Pérez-Castaño (2007) en la medición de la competitividad de las empresas se emplea el Business Competitiveness Index (BCI) propuesto por Michael Porter el cual hace uso de indicadores microeconómicos para la medición y evaluación del potencial productivo de una economía, que se manifiesta en la sofisticación de sus empresas y la calidad de su entorno microeconómico de negocios.

Las Pymes colombianas del clúster textilconfección, que para Castellanos et al. (2006, p. 38) es un sector de gran dinámica económica a nivel mundial, presentan debilidades estructurales e infraestructurales, que no les permiten alcanzar en el corto plazo altos niveles de competitividad acorde a las necesidades del mercado. De acuerdo con Giraldo-García (2008), Sarache-Castro (2003), Torres Acosta (2001) y CINTEX (2009), algunos aspectos que influyen negativamente en la competitividad de las Pymes colombianas son los siguientes:

Debilidades en el direccionamiento estratégico de la fabricación.

Baja capacidad administrativa para vincularse con el mercado internacional.

Sistemas de producción no alineados con las prioridades competitivas.

Ausencia de sistemas integrados de planeación, programación y control de la producción.

Deficiencias en los sistemas de costos de producción.

Desconocimiento de la capacidad de producción

Deficiencias en los sistemas de calidad.

Atraso y obsolescencia tecnológica.

Dificultad para incursionar en mercados sofisticados con productos más diferenciados.

Falta de una mejor comprensión de sus fortalezas competitivas y debilidades.

Falta una actitud más agresiva hacia el aprendizaje y la modernidad institucional.
Las Pymes de confección en lo particular poseen una estructura plana en la cual el gerente (en muchos casos propietario) centra su actividad en desarrollar funciones del nivel operativo, descuidando decisiones concernientes al nivel estratégico y táctico, según lo reporta el estudio hecho por Sarache-Castro (2003). La industria textil y de confección, se ha destacado como factor fundamental en el desarrollo del país, ya que durante más de 80 años ha hecho una contribución muy importante al crecimiento de las exportaciones y del PIB de la nación. Es de destacar que la industria de confecciones creció $11.8 \%$ en términos reales (precios del 2000) entre el 2006 y 2007, según Proexport (2009).

Según información de Inexmoda, Colombia es reconocida internacionalmente como un país que presenta grandes fortalezas en el negocio de los textiles y confecciones y en particular, en el de la moda. Sin embargo, algunos subsectores de textiles y confección durante el período enero - junio del 2009 decrecieron (INEXMODA, 2009). La Tabla 1 muestra los valores de las exportaciones de confecciones colombianas por tipo de tejido para los años 2008 y 2009, con un valor total exportado aproximado de 1800 millones de dólares. Colombia presenta uno de los costos laborales más bajos de Latinoamérica siendo la flexibilidad laboral y la disponibilidad del recurso humano calificado los que pueden permitir que los proyectos productivos sean rentables en Colombia, (Proexport, 2009).

Tabla 1. Exportaciones de confecciones colombianas por tipo de tejido. Fuente: Inexmoda (2009).

\begin{tabular}{ccc}
\hline Subsector & \multicolumn{2}{c}{ FOB US\$ } \\
& $\mathbf{2 0 0 8}$ & $\mathbf{2 0 0 9}$ \\
\hline Punto & $588,809,095$ & $127,405,857$ \\
Plano & $603,248,376$ & $254,046,320$ \\
Ropa de hogar & $139,908,062$ & $46,172,498$ \\
Total Confecciones & $1,331,965,534$ & $427,624,677$ \\
\hline
\end{tabular}

Enmarcado en los anteriores aspectos del sector, se puede concluir que existe un gran potencial en vía de desarrollo, siendo el crecimiento y la evolución factores que han superado todas las expectativas, donde no propiamente han estado acompañadas de una adecuada estrategia 
operacional como marco de mejoramiento, sino por el contrario han estado guiadas de manera empírica según Trujillo-Cabrera (2005), sin tener en cuenta que "el éxito comercial para una organización depende de una ventaja en el costo o de una ventaja en el valor" [Ohmae (1982) y Porter (1991) citados por Sarache-Castro (2003) y Sarache- Castro et al. (2004)].

A nivel microeconómico, el procedimiento validado por Sarache-Castro et al. (2007) en Pymes del sector metalmecánico y con posibilidad de aplicación en otros sectores, propone un esquema de mejoramiento coherente en tres aspectos fundamentales, denominado conjuntamente P-S-P: 1) las prioridades competitivas $(\mathrm{P}), 2)$, la configuración productiva o sistema de producción (S), y 3), las palancas de fabricación $(\mathrm{P})$. Esto significa que una acción de mejora en un sistema de producción debe partir por identificar las prioridades competitivas o exigencias del mercado objetivo; a partir de éstas, se debe valorar la coherencia de la configuración adoptada por el sistema productivo y el desempeño de sus subsistemas o palancas de fabricación con dichas prioridades competitivas, según se expresa en Giraldo et al. (2010). Para Krajewski et al. (2008), las prioridades competitivas son “... las dimensiones operativas cruciales que un proceso debe poseer para satisfacer a los clientes internos y externos, tanto en el presente como en el futuro". A nivel de estrategia de producción, autores como Miltenburg (2004) e Ibarra (2003) recalcan la necesidad de medir la mejora en un sistema de producción en términos de múltiples prioridades competitivas (múltiples criterios), en razón a que éstas últimas están relacionadas de manera más natural con un mercado objetivo. El estado del arte permite constatar la existencia de seis prioridades competitivas básicas en producción: calidad, costo, entrega, innovación, flexibilidad y servicio [Gaither \& Frazier (2000) e IbarraMirón \& Sarache-Castro (2008), Miltenburg (2004)].

De otra parte, para Schroeder (2004), la administración de operaciones tiene relación con la toma de decisiones sobre cuatro cuestiones relevantes: localización, proceso, capacidad y distribución física, siendo estas dos últimas las que dan a lugar a lo que se denomina la configuración productiva del sistema. Diferentes autores, tales como Domínguez Machuca et al. (1995), Schroeder (2004), Alfalla et al. (2008), entre otros; han reconocido la existencia de 4 tipos de configuraciones productivas genéricas que son: configuración por proyecto, configuración orientada al proceso o funcional (job-shop), la configuración orientada al producto (flow-shop) y configuraciones híbridas. La estructura del sistema de producción comprende, por un lado, al subsistema virtual y de decisión (o de gestión), responsable de la fijación de una estrategia funcional y de las funciones de planificación, programación y control; por otro lado, está el subsistema físico (o de transformación), conformado por un conjunto de personas, máquinas, materiales e instalaciones que en general se encargan de ejecutar el programa de producción, bajo algún esquema de organización productiva.

Definir cuál es la configuración productiva más adecuada para una empresa representa un compromiso fundamental en el establecimiento de la estrategia corporativa. Es decir, como lo señala Domínguez Machuca et al. (1995), estas son decisiones de diseño que pertenecen al nivel estratégico del esquema de administración de la producción y que están en relación con el nivel estratégico de la gestión corporativa. La selección de la configuración productiva depende de las prioridades competitivas que se persigan. Es decir, la configuración productiva a seleccionar o la mejora de una configuración existente, debe ser coherente con el output de fabricación que se desea proveer Miltenburg (2004), Ibarra-Mirón \& Sarache- Castro (2008). De modo que, la matriz producto - proceso se convierte en una herramienta para definir la estrategia de operaciones y por ende la ventaja competitiva que se desea alcanzar.

Algunos autores, establecen que debe existir una adecuada relación entre la configuración productiva y el cumplimiento de las prioridades y que éstas deben estar soportadas en los subsistemas del sistema de producción ó palancas de fabricación. Para Miltenburg (2004) las palancas de fabricación son: recursos humanos, estructura y controles de la organización, 
fuentes de aprovisionamiento, planificación y control de la producción, tecnología de procesos e instalaciones. En Giraldo-García (2008) se plantea que para medir el grado de efectividad de un sistema productivo por medio de las prioridades competitivas seleccionadas y jerarquizadas, se requiere intervenir factores como la configuración productiva que se adopte y las palancas de fabricación anteriormente mencionadas; sin embargo, realizar esta intervención en el sistema real puede resultar inapropiado, generar impactos costosos $\mathrm{y}$ traumáticos para la organización.

En Sarache-Castro et al. (2007) se propone que “... una vez se hayan estudiado con detalle los elementos del sistema P-S-P, es necesario analizar su coherencia estructural; es decir, qué tan alineados están el sistema de producción y sus palancas con las prioridades competitivas. Por ejemplo, si las prioridades competitivas más importantes son el costo y el plazo, el sistema de producción y las palancas deberán estar orientados hacia el logro de tales objetivos". Desde esta perspectiva, la adopción de prácticas encaminadas a la mejora continua de los procesos productivos se convierte en una de las más importantes tendencias para la administración moderna. Es por ello que se plantea la pregunta de investigación: ¿Cuál sería la estrategia operacional que se debe implementar en Pymes del sector confecciones para mejorar el desempeño de sus sistemas de producción frente a las prioridades competitivas de su mercado objetivo?

Por lo anteriormente expuesto, se pretende en este artículo mostrar los resultados obtenidos al hacer una investigación en Pymes de la confección textil sobre sus sistemas de producción y mercado objetivo. Estos resultados hacen referencia a presentar una metodología de mejoramiento del desempeño del sistema de producción consistente en la utilización de técnicas multicriterio para la selección y jerarquización de las prioridades competitivas a las que el sistema debe responder; el empleo de un procedimiento general para evaluar la coherencia estructural de la estrategia de operaciones en términos de las prioridades competitivas, los sistemas de producción y las palancas de fabricación; y finalmente la modelación del sistema de producción mediante simulación de eventos discretos con el fin de experimentar diversas alternativas de mejoramiento que estén alineadas con las prioridades competitivas más relevantes. Como resultado se aplicó la metodología propuesta a una muestra de cinco Pymes del sector de la confección de la ciudad de Manizales (Colombia) en su primera etapa, encontrándose que las prioridades competitivas costo, calidad y tiempo de entrega son las más relevantes. Seguidamente a la Pyme con el peor desempeño se le aplicaron las demás etapas, demostrándose finalmente la utilidad de la metodología al lograr variar el desempeño de su sistema de producción en términos de aumentar un Indicador de Efectividad del sistema de producción.

\section{Metodología}

\subsection{Identificación de las prioridades competitivas (P) para las empresas objeto de estudio.}

En esta etapa se sugiere aplicar el procedimiento validado por Sarache et al. $(2005$, p. 8688), ajustado del sector metalmecánico al sector confección textil, el cual permite definir y jerarquizar el conjunto de prioridades competitivas que exige un mercado objetivo. Los pasos utilizados para realizar la jerarquización de las prioridades competitivas y sus dimensiones son:

a. Definir las prioridades fundamentales: consiste en detectar las exigencias de los clientes que conforman el mercado objetivo a través de encuestas, investigación de mercados, estudios de benchmarking, consulta con expertos, etc.

b. Definir las dimensiones para cada prioridad: se desagregan las necesidades de los clientes en términos de dimensiones cualitativas $\mathrm{y}$ cuantitativas.

c. Calcular el número de expertos: se hace mediante el empleo de fórmula expresada en términos del nivel de precisión deseado, porcentaje de error a tolerar y un nivel de confianza dado. 
d. Seleccionar expertos: se tiene en cuenta a los propios clientes del mercado objetivo, expertos conocedores del mercado objetivo (gremios, académicos, agencias gubernamentales), etc.

e. Jerarquizar prioridades y dimensiones: los expertos proceden a calificar la importancia relativa entre el conjunto de prioridades y entre las dimensiones de cada prioridad.

f. Realizar pruebas de concordancia: consiste en medir el nivel de acuerdo entre los expertos a través de un indicador de concordancia.

g. Construir el Indicador de Efectividad: se obtiene de expresión dada en términos de los pesos de las prioridades competitivas, pesos de las dimensiones y calificaciones dados por los expertos y/o gerentes de empresas y está dado por la Ec. (1):

$$
I E_{i}=\sum_{j=1}^{n} W_{i j} \sum_{k=1}^{l} W_{i j k} * C_{i j k}
$$

Dónde:

$\mathrm{IE}_{\mathrm{i}}$ : Indicador de Efectividad que alcanza una empresa i. Se da en una escala de 0 a 5

i: índice de empresa en estudio

$\mathrm{j}$ : índice de prioridad competitiva

k: índice de dimensión de prioridad

1: número de dimensiones de la prioridad $\mathrm{j}$

$\mathrm{W}_{\mathrm{ij}}$ : Peso de la prioridad $\mathrm{j}$ en la empresa $\mathrm{i}$ (calidad, precio, entrega, flexibilidad, servicio, etc.)

$\mathrm{W}_{\mathrm{ij} \mathrm{k}}$ : Peso de la dimensión $\mathrm{k}$ en el factor j para la empresa i

$\mathrm{C}_{\mathrm{ij \textrm {k }}}$ : Calificación obtenida por la dimensión $\mathrm{k}$, en la prioridad $\mathrm{j}$, para la empresa $\mathrm{i}$

Finalmente una vez definidas las prioridades competitivas, es necesario evaluar el desempeño de las empresas en cada una de estas y en cada una de sus dimensiones, con el fin de proceder a clasificarlas en función de sus tendencias, Sarache-Castro et al. (2007).

\subsection{Análisis del sistema P-S-P}

Consiste en diagnosticar y analizar de manera integral las prioridades competitivas $(\mathrm{P})$, la configuración productiva (S) y las palancas de fabricación (P) de la empresa en estudio, según Sarache et al. (2007). La información requerida para el diagnóstico se recolecta a través de un instrumento tipo encuesta. En este paso se desarrollan tres actividades: 1) desempeño en las prioridades competitivas, 2) caracterización de la configuración productiva y, 3) análisis de las palancas de fabricación.

\subsubsection{Desempeño en las prioridades} competitivas: En esta parte del diagnóstico, se mide el desempeño de las empresas en las prioridades competitivas y en cada una de sus dimensiones; así mismo, cuantificar el desempeño (indicador de efectividad: $\mathrm{IE}_{\mathrm{i}}$ ) de la empresa o conjunto de empresas. Es deseable que la medición de este desempeño la haga el respectivo grupo gerencial de cada empresa a través del mencionado instrumento de encuesta (autoevaluación).

\subsubsection{Caracterización de la configuración} productiva: Para realizar el diagnóstico de cada uno de los elementos que forman parte del sistema de producción, la recolección de información debe abordar diferentes aspectos, como son: el sistema de aprovisionamiento, el sistema de fabricación y el sistema de distribución, caracterizando estos sistemas en términos de si corresponden a configuración por proyecto, proceso o producto e identificar el tipo de sistema de producción que la empresa o empresas en estudio aplican, procurando detectar las características relevantes en términos de flexibilidad y productividad que ofrece el sistema empleado.

\subsubsection{Caracterización de las palancas} de fabricación: Incluye la valoración del conjunto de palancas de fabricación, es decir, recursos humanos, planificación y control de la producción, estructura y controles de la organización, los procesos y las instalaciones. El detalle de esta caracterización y el análisis de las palancas de fabricación permitirán establecer las variables de decisión que se tendrán en cuenta en la etapa de mejoramiento. 


\subsection{Valoración de la coherencia del sistema P-S-P}

Se hace una exploración de los elementos de la triada P-S-P, analizando qué tan relacionadas se encuentran las prioridades competitivas (P) (según la jerarquía obtenida en 2.2.1), el sistema de producción (S) y sus palancas de fabricación (P).

Como se mencionó anteriormente, cada tipo de configuración productiva o sistema de producción logra obtener en mayor o menor grado impactar a las prioridades competitivas. Por lo tanto, es necesario identificar el máximo nivel que logra obtener una prioridad competitiva con un sistema de producción determinado. Por ejemplo, según Miltenburg (2004), para lograr ser el mejor en flexibilidad, resulta más adecuado tener un sistema Job Shop, de igual manera, si se quiere tener costos más bajos, estos se pueden conseguir con un sistema continuo.

De igual modo la relación entre palancas de fabricación y prioridades competitivas se puede observar en que a medida que se interviene cada palanca, se puede conseguir impactos positivos o negativos en las prioridades, como por ejemplo, si centramos acciones inicialmente en la palanca recursos humanos, promoviendo la polivalencia de operaciones y el incremento de la eficiencia, se pueden lograr mejoras en costo y plazo de entrega.

En síntesis el resultado de esta etapa es formular un modelo de prescripción coherente del tipo Variables de Decisión: Medidas de Desempeño (VD:MD), para cada empresa en estudio en el cual las variables de decisión (VD) se corresponden con la configuración productiva y las palancas de fabricación, mientras que las medidas de desempeño (MD) se corresponden con las prioridades competitivas. La formulación de este modelo se hace en conjunto con el grupo gerencial de la empresa en estudio.

\subsection{Definición y evaluación de las acciones de mejoramiento}

Con base en la valoración de la coherencia del sistema P-S-P, el grupo gerencial define uno o varios escenarios basados en el modelo prescriptivo del tipo VD:MD y evalúa el impacto de las respectivas VDs en la(s) $\mathrm{MD}_{(\mathrm{s})}$ a través de un modelo de simulación de eventos discretos y un Indicador de Efectividad (IE). Se espera que el modelo VD:MD de mayor impacto en las prioridades competitivas más relevantes sea el que adopte la empresa en el mundo real por lo que en la definición del modelo se sugiere emplear VDs cuyos valores sean factibles de implantar por la empresa desde el punto de vista económico y técnico. En la construcción del modelo de simulación se utiliza la metodología universalmente aceptada en estudios de simulación de eventos discretos, Giraldo-García (2008).

\section{Resultados y discusión}

Se seleccionó de un universo aproximado de 20 empresas una muestra de cinco (5) Pymes de la confección ubicadas en la ciudad de Manizales (Caldas), aplicándose la metodología a todas ellas en la primera etapa (numeral 2.1). Posteriormente se eligió la Pyme con un IE (Indicador de Efectividad) bajo para aplicar las demás etapas. A pesar de que la metodología puede aplicarse a cualquier grupo de empresas de un sector, se mostrara aquí una pequeña aplicación al sector confecciones con el fin de motivar la extensión de su uso a un número mayor de Pymes del sector.

\subsection{Identificación de las prioridades competitivas para las empresas objeto de estudio.}

a. Definir las prioridades fundamentales: A partir de la propuesta teórica de Miltenburg (2004) sobre las prioridades competitivas a las que debe responder un sistema de producción y con base en las investigaciones de SaracheCastro (2003) y Sarache-Castro et al. (2011) realizada en el sector de las confecciones, se encontró que las principales prioridades que un proveedor confeccionista en el medio colombiano debe potenciar son: costo, calidad, entrega, flexibilidad y asistencia técnica.

b. Definición de las dimensiones para cada prioridad: Como parte adicional a las preguntas 
de la ronda anterior, Sarache-Castro (2003) cuestionó a cada uno de los representantes sobre cuál o cuáles serían los componentes o dimensiones de cada una de las prioridades que se definieron anteriormente. En la Tabla 2 se presentan los resultados obtenidos.

c. Calculo del número de expertos: En esta fase del proceso se aplicó la expresión propuesta por Sarache (2003) según se muestra en la Tabla 3, la cual arrojó el empleo de cinco (5) expertos.

d. Selección de expertos: En la Tabla 4 se expone el listado de los cinco expertos que aceptaron participar en el presente estudio.

e. Jerarquización de prioridades y dimensiones. La Tabla 5 muestra la jerarquía de prioridades competitivas y sus dimensiones, establecidas por los expertos. De acuerdo con los resultados de la Tabla 5, es importante resaltar que las prioridades competitivas y dimensiones $\mathrm{o}$ componentes más destacados son: calidad (la fiabilidad en la entrega), entrega (el tiempo de respuesta a un cliente que pide una cotización), costo (por ser de tipo unidimensional y de relación directa con el precio unitario) y flexibilidad (tamaño del lote).

f. Realizar prueba de concordancia: Los expertos procedieron a calificar la importancia relativa entre el conjunto de prioridades y entre las dimensiones de cada prioridad obteniéndose un indicador de concordancia de Kendall de 0.696, el cual garantiza la coherencia de los expertos, dado que es superior a 0.5 .

g. Construcción del Indicador de Efectividad (IE): La Ec. (2) corresponde a la formula obtenida de la Tabla 5 que se empleara para valorar el Indicador de Efectividad $\mathrm{IE}_{\mathrm{i}}$ para cada Pyme estudiada.

Tabla 2. Prioridades competitivas y sus dimensiones. Fuente: Sarache-Castro (2003)

\begin{tabular}{|c|c|}
\hline $\begin{array}{l}\text { Prioridad } \\
\text { Competitiva }\end{array}$ & Dimensión \\
\hline Costo & Sin componentes \\
\hline Calidad & $\begin{array}{l}\text { 1. Fiabilidad en las entrega } \\
\text { 2. Calidad de concordancia } \\
\text { 3. Posibilidad de rechazo y } \\
\text { reclamaciones }\end{array}$ \\
\hline Entrega & $\begin{array}{l}\text { 1. Plazo de entrega } \\
\text { 2. Tiempo de respuesta a un } \\
\text { cliente que pide una cotización } \\
\text { 3. Tiempo de respuesta a un } \\
\text { cliente que pide información }\end{array}$ \\
\hline Flexibilidad & $\begin{array}{l}\text { 1. Flexibilidad ante cambios de } \\
\text { imprevistos } \\
\text { 2. Flexibilidad del portafolio de } \\
\text { familias de productos } \\
\text { 3. Flexibilidad en el tamaño de } \\
\text { los pedidos } \\
\text { 4. Flexibilidad del portafolio de } \\
\text { tejidos }\end{array}$ \\
\hline $\begin{array}{l}\text { Asistencia } \\
\text { técnica }\end{array}$ & Sin componentes \\
\hline
\end{tabular}

$$
\begin{aligned}
I E_{i}= & \left(0.38\left[0.35 \mathrm{C}_{\mathrm{i} 21}+0.14 \mathrm{C}_{\mathrm{i} 22}+0.51 \mathrm{C}_{\mathrm{i} 23}\right]+0.26\left[0.32 \mathrm{C}_{\mathrm{i} 31}+0.16 \mathrm{C}_{\mathrm{i} 32}+0.52 \mathrm{C}_{\mathrm{i} 33}\right]+0.19\left[\mathrm{C}_{\mathrm{i} 1}\right]+\right. \\
& \left.0.09\left[0.46 \mathrm{C}_{\mathrm{i} 41}+0.15 \mathrm{C}_{\mathrm{i} 42}+0.18 \mathrm{C}_{\mathrm{i} 43}+0.21 \mathrm{C}_{\mathrm{i} 44}\right]+0.08\left[\mathrm{C}_{\mathrm{i} 51}\right]\right) * 0.60
\end{aligned}
$$

\begin{tabular}{|c|c|c|c|}
\hline Formula & Dónde: & $\begin{array}{c}\text { Valores } \\
\text { asignados }\end{array}$ & $\begin{array}{c}\text { Cantidad de } \\
\text { Expertos }\end{array}$ \\
\hline & n: número de expertos & & \multirow{4}{*}{5 personas } \\
\hline \multirow{3}{*}{$n=\frac{P(1-P) K}{i^{2}}$} & i: nivel de precisión deseado & 17 & \\
\hline & como promedis se toler & 004 & \\
\hline & $\mathrm{K}$ : constante asociada al nivel de confianza & 3.8416 & \\
\hline
\end{tabular}

Tabla 3. Calculo número del número de Expertos. 
Tabla 4. Listado de Expertos.

\begin{tabular}{cccc}
\hline Experto & Empresa/Entidad & Área de trabajo & Formación Profesional \\
\hline E1 & Sena & Instructora de Confección & Técnico \\
E2 & Tropical Beach & Administrador de Producción & Ingeniero Industrial \\
E3 & C.I Nicole & Jefe de Ingeniería & Ingeniero Industrial \\
E4 & C.I Color Siete & Gerente Comercial & \\
E5 & C.I Indudise & Gerente General & Economista \\
\hline
\end{tabular}

Tabla 5. Jerarquización de prioridades competitivas para Pymes de la confección.

\begin{tabular}{|c|c|c|c|c|}
\hline $\begin{array}{l}\text { Prioridades y dimensiones en } \\
\text { orden de importancia }\end{array}$ & Peso & $\mathrm{C}_{\mathrm{jk}}$ & Dimensiones & $\begin{array}{l}\text { Peso entre } \\
\text { dimensiones }\end{array}$ \\
\hline \multirow{4}{*}{ CALIDAD } & \multirow{4}{*}{0.38} & $\mathrm{C} 21$ & Calidad de Concordancia & 0.35 \\
\hline & & $\mathrm{C} 22$ & Posibilidad de rechazo y reclamaciones & 0.14 \\
\hline & & $\mathrm{C} 23$ & Fiabilidad en la entrega & 0.51 \\
\hline & & $\mathrm{C} 31$ & Plazo de entrega & 0.32 \\
\hline \multirow[t]{2}{*}{ ENTREGA } & \multirow[t]{2}{*}{0.26} & $\mathrm{C} 32$ & $\begin{array}{l}\text { Tiempo de respuesta a un cliente que pide } \\
\text { información }\end{array}$ & 0.16 \\
\hline & & $\mathrm{C} 33$ & Tiempo de respuesta a un cliente que pide cotización & 0.52 \\
\hline \multirow[t]{2}{*}{ PRECIO } & \multirow[t]{2}{*}{0.19} & $\mathrm{C} 11$ & Sin componentes & 1.00 \\
\hline & & $\mathrm{C} 41$ & En el tamaño del lote & 0.46 \\
\hline \multirow{3}{*}{ FLEXIBILIDAD } & \multirow{3}{*}{0.09} & $\mathrm{C} 42$ & Ante cambios de imprevistos & 0.15 \\
\hline & & $\mathrm{C} 43$ & Del portafolio de familias de productos & 0.18 \\
\hline & & $\mathrm{C} 44$ & Del portafolio de tejidos & 0.21 \\
\hline ASISTENCIA TÉCNICA & 0.08 & $\mathrm{C} 51$ & Sin componentes & 1.00 \\
\hline
\end{tabular}

\subsection{Análisis del sistema P-S-P}

\subsubsection{Desempeño en las prioridades competitivas}

La medición de este desempeño la hizo el respectivo grupo gerencial de cada empresa a través del instrumento de encuesta (autoevaluación) empleado y aplicado por Solano (2010). Tomando esta medición y aplicando la Ec. (2) se obtiene para cada empresa en estudio su respectivo Indicador de Efectividad (IE) según se muestra en la Tabla 6. Debe connotarse que las Pymes 1 y 3 presentan un $\mathrm{IE}_{\mathrm{i}}$ entre 2 y 3 que corresponde a un desempeño medio bajo y la Pyme 4 con $\mathrm{IE}_{\mathrm{i}}$ por debajo de 2 muestra un desempeño bajo según escala mostrada en Sarache-Castro (2003).

Para la aplicación de la metodología de mejoramiento en los siguientes pasos y etapas se empleará la información de la Pyme 1 la cual empezó a operar en marzo del año 2001, con ventas a nivel nacional e internacional, siendo sus productos reconocidos por ser confeccionados cumpliendo altos estándares de calidad en cuanto a materias primas e insumos. Cuenta con alrededor de 40 empleados en temporada baja y 100 en temporada alta. Por el particular interés de la empresa de entrar a participar en el mercado nacional e internacional adquiere nueva maquinaria que responde a la acelerada evolución que en su momento se da en la comercialización de ropa interior, exterior, casual y deportiva. La empresa aspira a ocupar uno de los puestos destacados en la industria de la región, del país y del mercado americano. Desde el año 2001 ha realizado exportaciones a países como: Puerto Rico, Panamá, México, Estados Unidos, Ecuador y Venezuela; actualmente su mayor mercado se ha canalizado hacia Venezuela exportando alrededor de 800.000 unidades al año. 
Tabla 6. Indicador de efectividad para 5 Pymes de confección de Manizales.

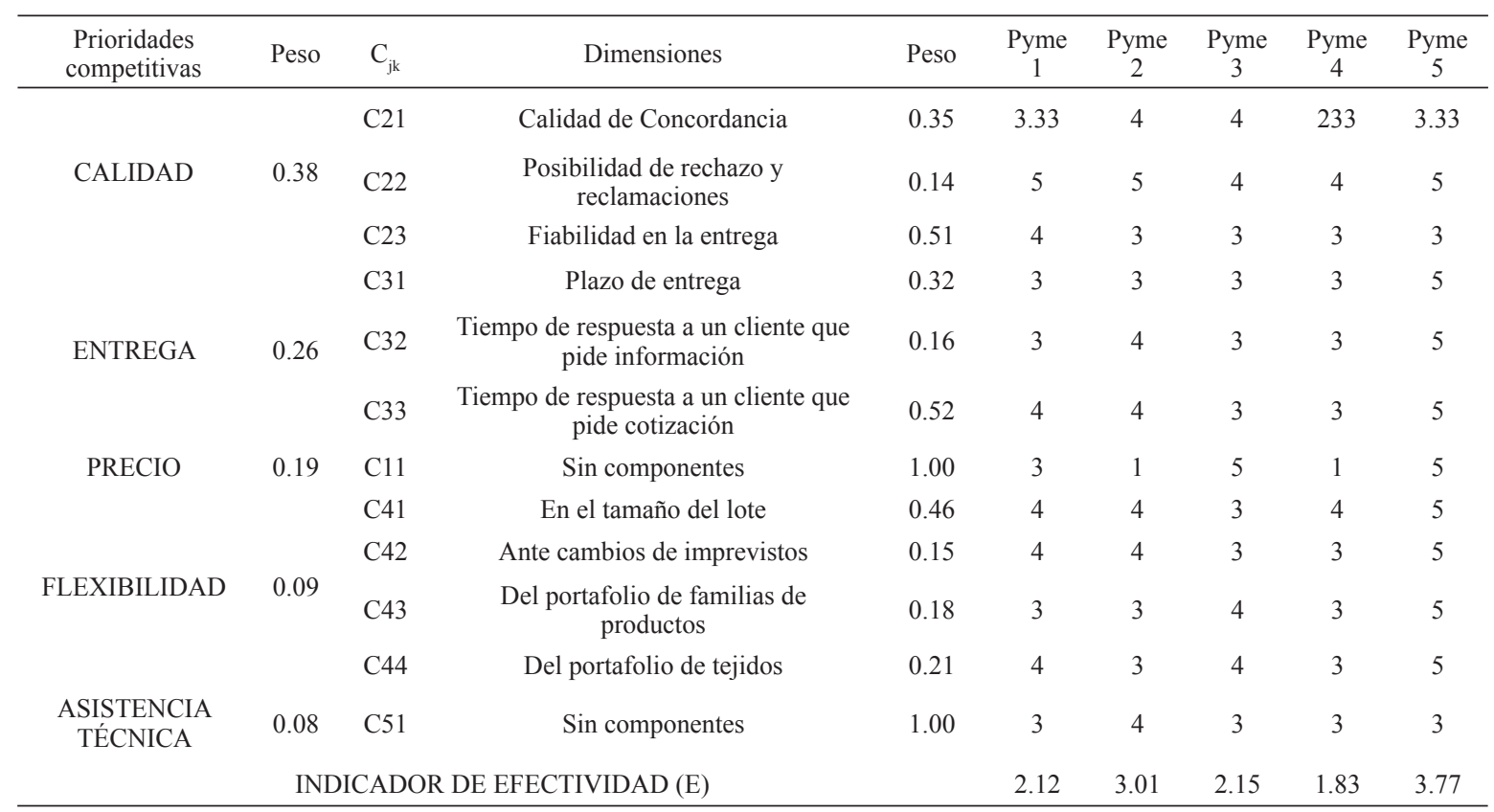

\subsubsection{Caracterización de la configuración productiva}

El sistema productivo de esta empresa corresponde a un Job Shop dedicado a la producción de sus propias colecciones y en algunos casos presta servicios de maquila. El módulo de producción que se simulará cuenta con 12 máquinas discriminadas así: 7 fileteadoras, 2 collarines, 2 planas, 1 cerradora de codo y 1 estación de inspección. Tiene mediana flexibilidad, sus diseños en gran parte son propios, y en el caso de la maquila se acogen a los diseños del cliente. El $35 \%$ de su producción se destina al mercado nacional y el $65 \%$ es para exportación. Posee instalaciones propias, en un lugar de fácil acceso para la recepción de materias primas y despacho de productos terminados. La producción se planea y realiza en tiempos cortos, a veces inferiores a una semana empleando un solo turno de trabajo. Las ventas de la empresa se hacen a través de un grupo de vendedores y un punto de venta propio, el segmento de mercado que atiende es estrato medio-bajo siendo los mejores meses para las ventas: junio, octubre, noviembre y diciembre. Más de la mitad de sus clientes son internacionales.

Posee la Pyme un sistema computarizado de registro y control de inventarios para todo el proceso productivo, desde la materia prima, hasta el producto terminado; las compras se realizan al comienzo de la producción; la mitad de los proveedores son de la ciudad y/o región y la otra mitad son del resto del país. En las líneas de producción se presenta un alto nivel de inventario en proceso.

Los controles de calidad en la empresa se aplican en todo el proceso, se buscan defectos en la medida, imperfectos en la tela, defectos en el corte, acabados o costuras utilizando un método de muestreo aleatorio. Pero cabe aclarar, que poseen deficiencias en la recolección de datos. No se cuenta con el personal idóneo para liderar procesos de aseguramiento de calidad; se limitan solamente a realizar la inspección de productos terminados, sin tener un método y criterios estandarizados. El mantenimiento lo realiza personal de la empresa, clasificado en preventivo y correctivo. Es importante mencionar, que se presenta con mayor frecuencia el mantenimiento correctivo.

\subsubsection{Caracterización de las palancas de fabricación}

Las características esenciales de estas palancas se obtuvieron a través del instrumento de encuesta aplicado por Solano (2010). En 
relación con recursos humanos, en estas Pymes se encuentra trabajando algún miembro de la familia, sobre todo en cargos directivos. El nivel educativo que tiene el personal se encuentra dividido en $4 \%$ técnico y tecnológico, $90 \%$ secundario y $6 \%$ estudios superiores concluidos. El $70 \%$ del personal que labora en la empresa se encuentra en producción y el $30 \%$ en el área administrativa. Se presenta dificultad para conseguir personal calificado y especializado que soporte la labor administrativa. Respecto a estructura y controles de la organización, se tienen formuladas la misión, visión y políticas, pero estás, no son entendidas y apropiadas en los niveles táctico y operativo, igualmente, no poseen sistemas de calidad certificados. No existen controles en los procesos. Su estructura jerárquica es de 2 niveles: Gerencia General y Administración.

Para planear la producción se cuenta con un procedimiento sistemático entre 1 y 3 meses, el cual es elaborado en coordinación con la gerencia, producción y ventas con base en estimaciones aproximadas, los pedidos en firme $(50 \%)$ y proyección estadística a partir de datos históricos $(50 \%)$. No se aplican herramientas informáticas para la gestión de la producción presentando dificultad para calcular y planear la capacidad de la planta.

Respecto al aprovisionamiento, la empresa en mención utiliza un sistema de control de inventarios para todos los procesos involucrados como son: bodegas de materia prima e insumos, producto en proceso y producto terminado. No se cuenta con una metodología para establecer la cantidad adecuada de compra de materiales siendo está influenciada por el juicio del gerente y necesidades de un programa de producción. En relación con la tecnología de procesos la empresa posee equipos semi-automatizados y automatizados.

\subsection{Valoración de la coherencia del sistema P-S-P}

Para la empresa objeto de estudio y basados en la Tabla 6, las siguientes prioridades competitivas deben tener un alto desempeño y cumplimiento: costo, calidad y entrega, pues recogen el $83 \%$ de la importancia relativa. Esto, sin duda, sin descuidar la flexibilidad y la asistencia técnica, ya que estas últimas pueden ir ascendiendo en un futuro cercano. Cabe mencionar, que de acuerdo a Miltenburg (2004), la prioridad que es coherente con la configuración productiva (Jop Shop) y que alcanza mejor desempeño es la flexibilidad, y la peor el costo, por lo que para el caso se percibe que no existe coherencia entre las prioridades y la configuración. El análisis de las palancas de fabricación muestra debilidades en algunas de ellas, como son: falta de uso de metodologías y herramientas informáticas para tener una planeación más adecuada; la existencia de un alto grado de participación familiar en la función directiva no permite una delimitación en las funciones y responsabilidades en especial el cargo de dirección de producción, ocasionando sobrecarga de actividades y descuido de las funciones propias del área o proceso.

En síntesis, para poder incrementar el desempeño en las prioridades competitivas más importantes (calidad, entrega y costo) para la Pyme y en común acuerdo con la gerencia se deben realizar acciones de mejora en: reducción de costos, mejoramiento de calidad, reducción de ciclos de fabricación y plazo de entrega. Inicialmente, es preciso intervenir el sistema físico de transformación mediante un cambio en la distribución en planta con el objetivo de mejorar su desempeño, es decir, mejorar la capacidad productiva con los recursos disponibles. En el caso de la calidad, la solución implica bajar la tasa de defectuosos. Y para el caso de las entregas, se hace necesario el desarrollo de un programa de mejoramiento de la eficiencia del módulo de confección.

Por tanto el modelo de prescripción coherente del tipo Variables de decisión: Medidas de desempeño ( $\left.\mathrm{VD}_{\mathrm{s}}: \mathrm{MD}_{\mathrm{s}}\right)$, que se propone, en conjunto con el grupo gerencial de la empresa, se estructuró así:

\section{Variables de decisión (VD):}

-Número y tipo máquinas en el módulo real de confección.

-Propuestas de distribución en planta.

-Numero de operarios.

-Tasa de defectuosos a alcanzar. 
Medidas de desempeño $\left(M D_{s}\right)$ :

-Costo

-Calidad

-Entrega

\subsection{Definición y evaluación de las acciones de mejoramiento}

El grupo gerencial estableció los siguientes cuatro (4) escenarios con base en el modelo prescriptivo propuesto en la etapa anterior:

Escenario 1: Representación actual del sistema de producción con su actual distribución en planta, máquinas y operarios y contemplando un $3 \%$ de producto no conforme o defectuoso y una tasa de rechazo total del $1 \%$. Este escenario se usa solo con fines de validación del modelo de simulación.

Escenario 2: Propuesta de mejora con cambio en la distribución en planta, retirando 1 máquina fileteadora e ingresando 1 máquina collarín para apoyar el flujo de producto en la operación cuello de botella e ingreso de 1 persona adicional para la operación de revisado. Se contempla como \% de producto no conforme o defectuoso del $2 \%$ y el rechazo total del producto del $0.5 \%$.

Escenario 3: Propuesta de mejora con cambio en la distribución en planta, se utilizan tiempos de operación de la empresa líder de la región (benchmarking), retiro de 2 fileteadoras e ingresando 1 maquina collarín para la operación cuello de botella, adicionalmente se ingresa 1 persona para el revisado. Se contempla como \% de producto no conforme o defectuoso del $2 \%$ y el rechazo total del producto del $0.5 \%$.

Escenario 4: Propuesta de mejora con cambio en la distribución en planta, retiro de 2 fileteadoras en la operación de pegado de mangas y para la operación de revisión se ingresa 1 persona adicional. Se contempla como $\%$ de producto no conforme o defectuoso del $2 \%$ y el rechazo total del producto del $0.5 \%$.

Los modelos de simulación de eventos discretos y sus escenarios se construyeron basados en los parámetros de operación que se muestran en la Tabla 7. La validación de los modelos de simulación se hizo comparando la cantidad de blusas producidas (tanto en la simulación del escenario 1 como en el sistema real) mediante el uso de la prueba $t$ según anexo 6 contenido en Solano (2010).

A partir de una longitud de corrida de 11 días con un turno de trabajo en el horario 7 am a 5:30 pm y simulando a 27 réplicas, según cálculos obtenidos por Solano (2010), para cada uno de los 4 escenarios se obtuvieron los resultados mostrados en la Tabla 8.

Para fines de interpretación de los datos de la Tabla 8, tener presente que:

El costo unitario de cada blusa es de $\$ 455$ (recordar que el cliente coloca materiales, diseños, etc.).

Productividad del factor trabajo $=$ Total producción / Total horas trabajadas.

El porcentaje de incremento en unidades se realiza con respecto al total producido real, es decir, 9478 unidades.

El costo unitario de producción incluye solo mano de obra y gastos generales de fabricación.

Al hacer un comparativo en términos de desempeño de cada escenario en la Tabla 8 se deduce que el mejor escenario es el 3. Respecto a la pregunta: ¿Cuáles serían las prioridades competitivas que se estarían impactando positivamente con el escenario 3 , en pro de la mejora del desempeño del sistema productivo?, tendríamos:

Calidad: con 0,41 unidades rechazadas, que corresponde menos del $1 \%$ de la producción total.

Entrega: obtención de un ciclo promedio de fabricación con un valor de 2,87 minutos por prenda ( 8 días de plazo de entrega), frente a 3,60 (9) y 5,07 (11) de los escenarios 2 y 4 respectivamente.

Costo: se obtuvo un costo unitario según la mano de obra utilizada de $\$ 277$, frente a $\$ 323$ y $\$ 324$, de los escenarios 2 y 4 respectivamente. 
Tabla 7. Datos generales empleados en construcción de los modelos de simulación.

\begin{tabular}{|c|c|}
\hline Referencia a modelar: & Blusa Bebita \\
\hline Cantidad de la referencia & 9478 unidades en 10 colores \\
\hline \multirow[t]{6}{*}{ Máquinas en el módulo real de confección } & 12 discriminadas así: \\
\hline & 7 Fileteadoras \\
\hline & 2 Collarín \\
\hline & 2 Planas \\
\hline & 1 Cerradora de Codo \\
\hline & 1 Estación de Revisión \\
\hline Tiempo real de la prenda & 4.15 MIN \\
\hline Numero de operarios sistema actual & 13 \\
\hline Periodo de fabricación & Dic 1 a dic al 11 de 2010 \\
\hline Diagrama de flujo del producto. & Ver Tesis de Maestría Solano (2010) \\
\hline Locaciones de procesamiento y almacenaje & Ver Tesis de Maestría Solano (2010) \\
\hline Secuencias de enrutamiento para cada entidad & Ver Tesis de Maestría Solano (2010) \\
\hline \multirow[t]{2}{*}{ Tipos de materia prima (entidades) necesarias para la fabricación. } & Ver Tesis de Maestría Solano (2010) \\
\hline & 7: 00 A.M - 5:30 PM \\
\hline \multirow[t]{2}{*}{ Horario de trabajo y descansos del personal } & $15 \mathrm{MIN}$ de descanso \\
\hline & $30 \mathrm{MIN}$ de almuerzo \\
\hline \multirow[t]{2}{*}{ Tiempos requeridos por cada entidad. } & Ver Tesis de Maestría Solano (2010) \\
\hline & Fileteadora: $1.5 \mathrm{MIN}$ \\
\hline \multirow{3}{*}{ Tiempos relacionados con los alistamientos en las máquinas } & Collarín: $1.5 \mathrm{MIN}$ \\
\hline & Plana: $1 \mathrm{MIN}$ \\
\hline & Cerradora de Codo: $2 \mathrm{MIN}$ \\
\hline Información de unidades defectuosas para el sistema en general & $\begin{array}{l}3 \% \text { de producto no conforme y } 1 \% \\
\text { rechazado }\end{array}$ \\
\hline Numero de replicas & 27 (Ver Tesis de Maestría Solano (2010)) \\
\hline
\end{tabular}

Tabla 8. Desempeño del sistema de producción para 4 escenarios simulados.

\begin{tabular}{|c|c|c|c|c|}
\hline Parámetros & $\begin{array}{c}\text { Escenario } \\
1 \\
\end{array}$ & Escenario 2 & $\begin{array}{c}\text { Escenario } \\
3 \\
\end{array}$ & $\begin{array}{c}\text { Escenario } \\
4 \\
\end{array}$ \\
\hline Día en que termina la producción & 11 & 9 & 8 & 11 \\
\hline Producción total (unidades) & 9,990 & 13,793 & 14,994 & 10.804 \\
\hline Producción promedio por día (unidades) & 768.52 & $1,045.42$ & 1,153 & 831.11 \\
\hline Ciclo total promedio (minutos) & 6.91 & 3.60 & 2.87 & 5.07 \\
\hline Productividad del factor trabajo (blusas) / (hora) & 6.65 & 9.18 & 9.98 & 7.19 \\
\hline Tiempo promedio en el sistema (horas) & 89.67 & 80.63 & 74.58 & 89.09 \\
\hline Tasa de rechazo promedio $(\%)$ & 0.25 & 0.67 & 0.41 & 0.32 \\
\hline Costo unitario & 384.7 & 323.67 & 277.0 & 324.72 \\
\hline Cumplimiento de la meta de producción (\%) & 76.85 & 104.54 & 115,3 & 83.11 \\
\hline Porcentaje de incremento en unidades & $5.40 \%$ & $45.52 \%$ & $58.20 \%$ & $14 \%$ \\
\hline Facturación de la producción adicional ( $\$ 455$ x unidad) & $\$ 232,960$ & $\$ 1,963,325$ & $\$ 2,509,780$ & $\$ 603,330$ \\
\hline
\end{tabular}


En el escenario 3 el total de producción lograda con base en los cambios de distribución en planta y adopción de tiempos de operación de una empresa líder de la región es de 14.494 unidades, las cuales se terminan de fabricar el día 8 a las 4:18 p.m. Esta reducción significativa del plazo de fabricación se debió a que en este escenario se separaron las operaciones del producto en dos, es decir, operaciones de iniciación y de ensamble, en la primera etapa, y en la segunda etapa se involucran operaciones de mayor rendimiento, que para el caso de la blusa "Bebita" son las operaciones de pegar, cerrar y dobladillar, las cuales se realizan en locaciones que desarrollan alta velocidad, logrando así un mejor flujo del material.

Igualmente en este escenario, con base en los resultados de tasas de utilización de centros de trabajo, se da el mayor porcentaje (promedio $68 \%$ ) de utilización en: collarín 3, revisado 1,2 y fileteadora 3 y 4 , donde se realizan las operaciones de: dobladillo, inspección 1 y 2 , cerrar lados, respectivamente. Adicionalmente se encuentran 4 locaciones que presentan un valor promedio del $31 \%$ de utilización, las cuales son: cerradora de codo, collarín 1 y 2 , plana 1, donde se realizan las operaciones de: pegar cinta al cuello, sesgar manga, pegar sesgo al cuello y por último zurcir mangas. Igualmente este escenario da la mejor tasa de productividad del factor trabajo: 9,98 blusas/ hora y un incremento en unidades producidas cercano al 58\%.

Finalmente la valoración de los impactos en el Indicador de Efectividad (IE) de la empresa, se realizó nuevamente revisando todas las prioridades y dimensiones. Este proceso fue ejecutado por la Gerencia General de la empresa objeto de estudio con el apoyo de la escala de Saaty (ver anexo 1 en Solano (2010)). En la tabla 9 se presenta la proyección de impactos en las prioridades competitivas. De acuerdo a los resultados obtenidos en la Tabla 9, se puede visualizar que el Indicador de Efectividad de la empresa pasó de un valor de 2,12 a un valor de 2,32 lo que representa un incremento del $10 \%$. Aunque el incremento del IE para la empresa es bajo, este resultado no se puede despreciar. Además de implementar el escenario 3 en el sistema real, el IE se puede seguir mejorando si la empresa empieza a trabajar no solo en las prioridades que alcanzó un aumento, sino también en las palancas de fabricación en donde necesita enfocar sus esfuerzos: implementar un sistema de calidad, reducción de tiempos de operación, mejorar la programación de materiales y capacidad con la ayuda de herramientas informáticas como el MRP y CRP, las cuales constituyen un soporte al proceso de toma de decisiones para programar la producción y evitar realizar los montajes de pedidos empíricamente.

\section{Conclusiones}

La identificación de las prioridades competitivas que el mercado objetivo exige a las Pymes de la confección debe ser el punto de partida para que estas definan una estrategia coherente e integral de mejoramiento de sus sistemas de producción. Coherente en el sentido que cualquier acción de mejoramiento que emprendan conlleve al logro de metas que satisfagan las necesidades de su mercado objetivo, sea este nacional o internacional, e integral desde el punto de vista de evitar obtener solo mejoras locales o parciales.

Luego de tener claro cuáles son las prioridades competitivas, se debe establecer el nivel de desempeño de la organización frente a los clientes y frente a sus competidores, mediante la medición del indicador de efectividad (IE) propuesto, el cual constituye un aporte esencial para la identificación de las acciones que se deben adoptar para el mejoramiento del sistema productivo, con el fin de mejorar la posición competitiva en el mercado objetivo.

Según el grupo de expertos consultado, para que la Pyme de la confección estudiada pueda mantenerse en altos niveles de desempeño frente a su mercado objetivo, debe mejorar simultáneamente en las prioridades competitivas: calidad, costo y entrega, las cuales alcanzan a cubrir hasta un $80 \%$ de la importancia relativa. Se requiere específicamente mejoras en las dimensiones: calidad de concordancia, costo de maquilar y plazo en la entrega. 
Tabla 9. Nueva valoración del indicador de efectividad (IE) de la Pyme 1.

\begin{tabular}{|c|c|c|c|c|c|c|}
\hline $\begin{array}{l}\text { Factores y } \\
\text { componentes } \\
\text { en orden de } \\
\text { importancia }\end{array}$ & $\begin{array}{c}\text { Peso } \\
\text { entre } \\
\text { factores }\end{array}$ & $\mathrm{Cjk}$ & Dimensiones & $\begin{array}{l}\text { Peso entre } \\
\text { dimensiones }\end{array}$ & $\begin{array}{l}\text { Calificación } \\
\text { Inicial }\end{array}$ & $\begin{array}{l}\text { Calificación } \\
\text { proyectada }\end{array}$ \\
\hline \multirow{4}{*}{ CALIDAD } & \multirow{4}{*}{0.36} & $\mathrm{C} 21$ & Calidad de Concordancia & 0.35 & 3.33 & 3.67 \\
\hline & & $\mathrm{C} 22$ & $\begin{array}{l}\text { Posibilidad de Rechazo y } \\
\text { reclamaciones }\end{array}$ & 0.14 & 5 & 5 \\
\hline & & $\mathrm{C} 23$ & Fiabilidad en la entrega & 0.51 & 4 & 4 \\
\hline & & $\mathrm{C} 31$ & Plazo de entrega & 0.32 & 3 & 4 \\
\hline \multirow[t]{2}{*}{ ENTREGA } & \multirow[t]{2}{*}{0.27} & $\mathrm{C} 32$ & $\begin{array}{l}\text { Tiempo de respuesta a un } \\
\text { cliente que pide información }\end{array}$ & 0.16 & 3 & 3 \\
\hline & & $\mathrm{C} 33$ & $\begin{array}{l}\text { Tiempo de respuesta a un } \\
\text { cliente que pide una cotización }\end{array}$ & 0.52 & 4 & 4 \\
\hline \multirow[t]{3}{*}{ COSTO } & \multirow[t]{2}{*}{0.20} & $\mathrm{C} 11$ & Sin componentes & 1.00 & 3 & 4 \\
\hline & & $\mathrm{C} 41$ & En el tamaño del lote & 0.46 & 4 & 4 \\
\hline & \multirow{3}{*}{0.09} & $\mathrm{C} 42$ & Ante cambios de imprevistos & 0.15 & 4 & 4 \\
\hline \multirow[t]{2}{*}{ FLEXIBILIDAD } & & $\mathrm{C} 43$ & $\begin{array}{l}\text { Del portafolio de familias de } \\
\text { productos }\end{array}$ & 0.18 & 3 & 4 \\
\hline & & $\mathrm{C} 44$ & Del portafolio de tejidos & 0.21 & 4 & 4 \\
\hline \multirow[t]{2}{*}{$\begin{array}{l}\text { ASISTENCIA } \\
\text { TÉCNICA }\end{array}$} & 0.08 & C51 & Sin componentes & 1.00 & 3 & 3 \\
\hline & \multicolumn{3}{|c|}{ INDICADOR DE EFECTIVIDAD } & & 2.12 & 2.32 \\
\hline
\end{tabular}

El análisis de las empresas objeto de estudio, desde la óptica P-S-P, demuestra que no hay coherencia estructural entre las exigencias del mercado, el sistema de producción adoptado y la organización de las palancas de fabricación. Dicha situación refleja la ausencia de un enfoque estratégico para la manufactura en las empresas de confección estudiadas; seguramente un estudio en las demás funciones gerenciales podría reflejar resultados similares. El estudio de cada una de ellas permite observar en algunos casos la adopción o la aproximación a buenas prácticas de producción; sin embargo, no es una situación constante a nivel del sector, pues mediciones efectuadas a través de un Indicador de Efectividad muestran Pymes con desempeños medio bajos y bajos.

Experimentar soluciones de mejoramiento directamente en los sistemas físicos de transformación (SFTs), es costoso, demanda alto consumo de tiempo y específicamente para una Pyme, cuyos recursos son muy limitados, le resulta imposible servir de laboratorio de experimentación, dejando de lado su operación económica. Los aportes teóricos consultados apuntan a que en los últimos años, se ha dado un buen desarrollo en el diseño/ejecución de experimentos empleando simulación computarizada, con ventajas muy superiores en términos de costo y tiempo, respecto a la experimentación física.

\section{Bibliografía}

Alfalla L, R., García S, M. R., Garrido V, P., González Z, M. M., \& Sacristán D, M. (2008). Introducción a la Dirección de Operaciones Táctico-Operativa: Un enfoque Práctico. Editorial: Delta Publicaciones Universitarias. Madrid. España.

Castellanos, O., Jimenez, C., Sinitsyn, A., Montañez, \& V. M., Sinitsyn O. A. (2006). Análisis del desarrollo tecnológico en la aplicación de enzimas en la industria textil. Ingeniería y Competitividad, 8 (1), p. 37- 46.

CINTEX, Centro de Investigación e Innovación Textil. (2009). Caso de Innovación. Sector Textil, Diseño y Confecciones. http://www. forossemana.com/doc/Doc-1894_20091127.pdf 
Domínguez M, J. A., Álvarez G, M. J., Domínguez M, M. A., García G, S. \& Ruiz J, A. (1995). Dirección de Operaciones. Aspectos tácticos y operativos en la producción y los servicios. Editorial Mc-Graw Hill, Madrid

Gaither, N. \& Fraizer, G. (2000). Administración de la Producción. $8^{\mathrm{a}}$ Edición. Thompson Editores: México. D.F.

Giraldo G, J. A. (2008). Metodología soportada en simulación para el mejoramiento de sistemas de producción. Aplicaciones en Pymes metalmecánicas. Tesis de Doctorado. Doctorado en Ingeniería. Universidad Nacional de Colombia.

Giraldo G, J. A., Sarache C, W.A., \& Castrillón G, O. D. (2010). Metodología integral soportada en simulación para el mejoramiento de sistemas de producción Job Shop. Aplicaciones en pymes metalmecánicas. Ingeniería e Investigación. Vol. 30. No. 1. P. 97.

Ibarra M, S. (2003). Modelo y procedimientos para el análisis y proyección competitiva de Unidades Estratégicas de Fabricación en empresas manufactureras cubanas. Tesis Doctoral Universidad Central "Marta Abreu" de Las Villas. Santa Clara, Cuba.

Ibarra M, S. \& Sarache C, W. A. (2008). Gestión de la Producción: Una Aproximación Conceptual. Colectivo de Autores. $1^{\mathrm{a}}$. Edición. Editorial. Universidad Nacional de Colombia.

INEXMODA. El Sector Textily de la Confección Colombiano. http://www.inexmoda.org.co/ TextilConfecci\%C3\%B3n/ElsectorTextilydel aConfecci\%C3\%B3nColombiano/tabid/280/ Default.aspx

Krajewski, L., Ritzman, L. P., \& Malhotra, M. K. (2008). Administración de Operaciones: Procesos y Cadenas de valor. $8^{\mathrm{a}}$ Edición. Pearson Educación. México.

Miltenburg, J. (2004): "Manufacturing Strategy”. Productivity Press. Portly, Oregón.

Pérez-Castaño, B. J. (2007). Competitividad, desarrollo e ingeniería: algunas definiciones y reflexiones. En: Ingeniería y Competitividad, Vol. $9 \mathrm{~N}^{\circ}$ 1, p. 84-91.

PROEXPORT. (2009). Invierta en Colombia. Trabajo, Compromiso, Ingenio. Industria Textil y Confecciones. Septiembre. http://www. inviertaencolombia.com.co/.../textil.html

Sarache C, W. A. (2003). Modelo con Enfoque Estratégico y Procedimientos para Contribuir al incremento del nivel de desempeño de las Pymes de confección desde la función de producción. Aplicaciones en la Región del Tolima, Colombia. Tesis de Doctorado en Ciencias Técnicas. Universidad Central "Marta Abreu"de las Villas. Cuba.

Sarache C, W. A., Marrero D. F., \& Hernández P. G. (2004). Objetivos de la función de operaciones. Aportes a la industria de la confección colombiana. Revista Universidad EAFIT. Vol. 40. No. 133. P. 35 - 46.

Sarache Castro W. A., Cárdenas D., \& Giraldo García J. A. (2005). Procedimiento para la definición y jerarquización de prioridades competitivas de fabricación. Aplicaciones en las pymes de la industria metalmecánica, Revista Ingeniería y Competitividad, Vol. 7 \# 2, pp.: 8491

Sarache C, W. A., Cárdenas A, D. M., Giraldo G, J. A., \& Parra S, J. H. (2007). Procedimiento para evaluar la estrategia de manufactura: Aplicaciones en la industria Metalmecánica. Cuadernos de Administración. No.033. (Ene. Jun.) p.103 - 123.

Sarache C, W. A., Castrillon G, O. D. \& Giraldo G, J. A., (2011). Prioridades competitivas para la industria de la confeccion. Estudio de caso. Cuadernos de Administración. No.043. (Jul. Dic.) p. 89 - 110.

Schroeder, R. (2004). Administración de Operaciones. Casos y conceptos contemporáneos. $2^{\mathrm{a}}$. Edición. Editorial. McGraw-Hill Companies, Inc. México.

Solano, A. (2010). Modelo de mejoramiento en el desempeño del sistema de producción 
en una empresa perteneciente a la industria de la confección mediante simulación de eventos discretos. Tesis de Maestría en Ingeniería, Área de Énfasis: Ingeniería Industrial, Facultad de Ingeniería - Universidad del Valle, Santiago de Cali, Colombia.

Torres A, J. H. (2001). Procedimiento para la planeación agregada en la pequeña y mediana industria manufacturera. Aplicación al sector industrial colombiano. Tesis de Doctorado. Universidad Central "Marta Abreu" de Las Villas. Santa Clara, Cuba.

Trujillo C, F. (2005). Las pymes de confecciones textiles y los paradigmas de producción. Publicación Electrónica. http:// www. monografias.com. 